\title{
Estrategias de aprendizaje de los estudiantes de enseñanza media de establecimientos educacionales municipales de la provincia de Talca
}

\author{
Learning strategies of the \\ middle school students of municipal educational establishments \\ from the province of Talca
}

\author{
CARLOS GARCÍA SOTO \\ Licenciado en Psicología \\ Universidad Autónoma de Chile, Sede Talca, Chile \\ Programa de Salud Mental, Ilustre Municipalidad de San Javier, Chile \\ crlsjvrg@ hotmail.com

\section{KARLA SALINAS DUARTE} \\ Licenciada en Psicología \\ Universidad Autónoma de Chile, Sede Talca, Chile \\ Departamento de Atención primaria de Salud Comunal de Maule, Chile \\ ksalinas@csmaule.com
}

\section{RESUMEN}

Comprender como los estudiantes aprenden a aprender según edad, rendimiento académico, autoestima y motivación ocupa un lugar importante en el ámbito del estudio de las estrategias de aprendizaje en la psicología de la educación, sin embargo, conocer como dichas estrategias se expresan según la condición geográfica o el tipo de enseñanza ha sido relativamente abordado. El siguiente estudio tiene como objetivo describir las estrategias de aprendizaje de los estudiantes de tercer año medio de liceos municipales de la provincia de Talca según área geográfica (urbano-rural) y tipo de enseñanza (técnica, politécnica o científico humanista). Consiste en un estudio cuantitativo, de diseño transversal descriptivo. La muestra estuvo conformada por 13 liceos municipales con 364 estudiantes, de ellos 10 liceos pertenecen a zonas urbanas $(n=285)$ y 3 a zonas rurales $(n=79)$. Se aplicó el Inventario de Estrategias de Aprendizajes de Ronald Schmeck y se midieron variables como género, tipo de enseñanza y ubicación geográfica de la escuela. Los resultados indican que se evidenció que existen diferencias en las estrategias de 
aprendizajes para cada una de las variables. En cuanto al sexo, las diferencias significativas se manifiestan en las estrategias procesamiento elaborativo y estudio metódico, siendo el grupo femenino aquel que marca una diferencia mayor en ambas estrategias. En relación al área geográfica (rural-urbano) existen diferencias con respecto a la estrategia estudio metódico, mostrándose una mayor tendencia a utilizar esta estrategia en el grupo rural. En conclusión, generar antecedentes que pueden contribuir a ayudar y facilitar la búsqueda de nuevas tácticas educativas que propicien la asimilación del currículum en concordancia con las estrategias que poseen los estudiantes de enseñanza media según sus contextos geográficos, culturales y educacionales.

Palabras clave: estrategias de aprendizaje, educación secundaria, educación rural, adolescencia.

\section{ABSTRACT}

Understanding that students learn according to age, academic performance, self-esteem and motivation plays an important role in the field of the study of learning strategies in educational psychology, however, understanding such strategies is expressed through the geographical conditions or through the type of education, has been relatively addressed. The objective of the present study is to describe the learning strategies of third year students in public schools from the province of Talca according to geographical area (urbanrural) and type of education (technical, polytechnic or scientific humanist). This quantitative study consists of a descriptive transversal design. The sample consisted of 13 public schools with 362 students, 10 schools from the urban area $(n=285)$ and 3 schools $(n=79)$. Ronald Schmeck's Inventory of Learning Strategies was applied in addition to measuring variables such as gender, learning type, and the school's geographical location. The results indicate that an evident difference in learning strategies for each of the variables exist. Regarding gender, significant differences manifests themselves in elaborate processing strategies and methodical study, with the female group being the one that made a greater difference in both strategies. In relation to the geographical area, (rural-urban) differences exist regarding the methodical study strategy; showing greater tendency to use this strategy in the rural group. In conclusion, generating background that can contribute to help and facilitate the search for new educational tactics that facilitate the assimilation of the curriculum in accordance with strategies for students in high school, according to their geographical, cultural and educational contexts.

Key words: learning strategies, secondary education, rural education, adolescence. 


\section{INTRODUCCIÓN}

La necesidad de tomar en cuenta el conjunto de variables individuales que inciden en el desempeño escolar como la motivación, los conocimientos previos y estrategias de aprendizajes entre otras, forman parte del reto que se enfrentan en el marco del proceso de perfeccionamiento de las escuelas en la mayoría de los países (Pizano, 2004). Una forma de enfrentar este reto es enfocando la mirada hacia las estrategias de aprendizajes. Si bien existen diversas definiciones (Badenier, 2003; Bermeosolo, 2005; Cabrera y Fariñas, 2005), éstas se pueden entender como procedimientos 0 actividades que se escogen con el propósito de facilitar la adquisición, almacenaje y/o utilización de información o conocimientos; varían según la información que sea procesada a un nivel superficial o fonológico (repetición) o más profundo y semántico (elaboración y organización), existiendo, a parte, unas estrategias de apoyo relacionadas con los procesos motivacionales. Las estrategias de aprendizaje son un constructo y refleja un carácter multidimensional del proceso de adquisición de conocimientos en el escolar.

Según Guanipa y Mogollón (2006) señala la necesidad de enseñar a los estudiantes estrategias cognitivas de resolución de problemas, pero no solo reduciéndolas al puro procedimiento de posibilitar el aprendizaje, sino también de acceder al proceso de aprender a aprender.

Las estrategias de aprendizaje poseen rasgos comunes como: 1) hacen referencias a los procesos que están a la base de una tarea intelectual; 2) son habilidades de control y regulación sobre habilidades específicas; 3 ) se diferencian en los alumnos con éxito y sin éxito académico; 4) se perfeccionan en el contexto escolar; 5) tienen el carácter de intencional; 6) son flexibles y modificables; 7) se pueden entrenar; 8) crean las condiciones para que se crean aprendizajes significativos; 9) son herramientas para adquirir, procesar, recuperar y transformar la información (Badenier, 2003).

Toda estrategia ocurre asociada con otros procesos y recursos cognitivos que posee cualquier aprendiz, estos procesos son:

- Procesos cognitivos básicos: son todas aquellas operaciones y procesos involucrados en el procesamiento de la información, como la atención, percepción, codificación, almacenaje y recuperación. 
- Base de conocimientos: hace referencia al bagaje de conocimientos que se posee sobre distintos temas el cual está organizado en forma jerárquica constituida por esquemas.

- Conocimiento estratégico: este tipo tiene directa conexión con lo que se llama estrategias de aprendizaje; es decir el saber cómo conocer.

- Metacognitivos: hace referencia al conocimiento que se posee sobre qué y cómo se sabe, así como del conocimiento que se posee de los propios procesos y operaciones cognitivas cuando se aprende, recuerda 0 se solucionan problemas.

Estos cuatro tipos de conocimientos interactúan en forma intrincada y compleja cuando el aprendiz utiliza las estrategias de aprendizaje (Zulia, 2006).

Cuando se aprende algo también se puede elegir entre distintos métodos y sistemas de aprendizaje. Dependiendo de lo que se quiera aprender, habrá interés en utilizar unas estrategias y no otras. No existen estrategias buenas y malas en sí mismas, pero sí estrategias adecuadas o inadecuadas para un contexto determinado. Los resultados que se obtienen, lo bien o rápido que se aprende dependen en gran medida de saber elegir la estrategia adecuada para cada tarea. Por lo tanto un estudiante emplea una estrategia cuando es capaz de ajustar su comportamiento a las exigencias de una actividad dada por el profesor y en las circunstancias en que esa tarea se produce. (Zulia, 2006).

De acuerdo a lo anterior, el modelo propuesto por Ronald Schmeck (1982, citado en Cabrera \& Fariñas, 2005), el cuál se vincula directamente a estudios sobre estrategias de aprendizaje, define tres estilos, cada uno supone el uso de una estrategia de aprendizaje particular por el estudiante.

- El estilo de profundidad: propio de aquel alumno que usa la estrategia de conceptualización, lo cual quiere decir que cuando estudia abstrae, analiza, relaciona y organiza las abstracciones.

- El estilo de elaboración: el cuál implica la utilización de una estrategia personalizada lo cual significa que el contenido de estudio ha de estar relacionado directamente con él mismo. 
- El estilo superficial: el cuál implica el uso de una estrategia centrada en la memorización.

Este modelo incorpora a la memoria en el proceso de aprendizaje, cuando una información se procesa profunda y elaborativamente, ésta se recuerda mejor, ya que el pensamiento profundo implica mayor dedicación al significado y clasificación de un pensamiento propuesto por un símbolo más que al símbolo mismo (Salinas 2004), de esta forma la inversión de tiempo es mayor y el cambio de conducta esperado de un aprendizaje perdura a largo plazo.

Los estudiantes que adoptan un estilo profundo, emplean más tiempo en el estudio y consideran el material que aprenden como más fácil de comprender que los estudiantes que adoptan un estilo superficial; estos al concentrarse en estrategias de memorización y retención, encuentran el trabajo pesado e ingrato y suelen fallar más frecuentemente en los exámenes (Cárdenas, 2006).

Por otro lado, Numerosos estudios, han aportado datos referente a la relación de estrategias de aprendizajes y con el rendimiento académico, autoestima, motivación y otras variables (Badenier, 2003; Massone y González, 2003; Muñoz, 2005; Salinas, 2004; Roux y Anzures, 2014; Zamora, Rubilar y Ramos, 2004). Un estudio realizado por Cano (2000) acerca de las estrategias de aprendizajes, demuestra la relación significativa entre la variable género de los estudiantes y sus despliegues de estrategias/estilos de aprendizajes, así como la influencia moduladora ejercida por una variable contextual y el tipo de carrera estudiada.

Otra investigación realizada por Gázquez, Pérez, Ruiz, Miras y Vicente (2006) con estudiantes de enseñanza secundaria muestra que no se evidencia relación significativa entre puntuaciones altas en autoestima y utilización o selección de estrategias de aprendizajes.

Estas investigaciones ponen de manifiesto el interés por los factores influyentes en cualquier proceso de aprendizaje, del mismo modo constituyen material empírico para dilucidar qué variables son importantes de tomar en cuenta para planificar programas educativos y evaluativos que generen una modificación sustancial en los estudiantes. Para lograr el punto anterior en esta investigación se toma como referencia las estrategias utilizadas para procesar la información por los estudiantes ya que estas logran recoger, organizar y utilizar el conocimiento. 
La literatura muestra la existencia de variadas investigaciones en contextos educacionales diversos con respecto a las estrategias de aprendizajes, sin embargo los estudios referentes a la relación de las estrategias utilizadas y los tipos de enseñanzas que se entregan en establecimientos urbanos y rurales, son escasos. Entendiendo que los establecimientos rurales poseen características distintivas además de su realidad geográfica y cultural, como son el ser establecimientos pequeños, en su mayoría atendidos por uno, dos o tres profesores, los que trabajan en aulas multigrado (Carrasco, Stingo y Laval, 2001). A partir de esto, se propuso como objetivo describir las estrategias de aprendizaje de los estudiantes de tercer año medio de liceos municipales de la provincia de Talca según área geográfica (urbano - rural) y tipo de enseñanza (técnica, técnico agrícola, politécnica y científico humanista).

Según lo planteado anteriormente, con todo lo anterior cobra sentido este componente formativo en el marco de la educación chilena ya que ayuda a generar programas de intervención para la construcción del conocimiento desde las edades más tempranas pudiendo hacer una intervención cognitiva que estimula el proceso de aprendizaje del estudiante obteniendo así logros cognitivos significativos y que les permitan favorecer sus procesos de formación continua. Para asegurar esos aprendizajes efectivos, se busca fortalecer las capacidades de las comunidades educativas, teniendo como principio el derecho de las y los jóvenes a una educación de calidad, a la participación e inclusión juvenil independientes de los contextos socioculturales a los que pertenezcan.

\section{MÉTODO}

El estudio corresponde a una metodología cuantitativa, de diseño transversal descriptivo. De una población general de 3034 estudiantes de tercer año medio de liceos municipales de la provincia de Talca que están en 22 liceos se escogieron 13 liceos (10 urbanos y 3 rurales), de los cuales se seleccionó una muestra aleatoria de 364 estudiantes. Sus edades fluctúan entre los 15 y 20 años, cuya edad promedio es de 17 años. De ellos, 285 son de liceos urbanos $(78,3 \%$ ) y 79 pertenecen a liceos rurales $(21,7 \%)$.

Para la recolección de los datos se consultó a la Provincial de Educación de Talca donde se obtuvo la información general de los liceos. Para el acceso a los liceos, se tomó contacto con cada uno de ellos de forma personal durante el año 2007, llevando una carta de presentación de la universidad donde se desarrolla la 
investigación y un consentimiento informado dirigido al director y a los apoderados de cada establecimiento educacional. Posteriormente y ya con la autorización por parte de la dirección se asignó un día de disponibilidad para la aplicación del inventario en los terceros medios.

En los días de aplicación se presentó a cada estudiante un consentimiento informado y a quienes quisieran participar se les aplicó al inventario. Se realizó una pequeña introducción para dar a conocer los objetivos del estudio así como también explicar el contenido del inventario presentado, la duración en tiempo para contestar el inventario fue entre 20 y 45 minutos.

El instrumento que se utilizó es el Inventario de Estrategias de Aprendizaje de Ronald Schmeck que fue adaptado a la población chilena por Truffello y Pérez (1998). Este inventario está compuesto por 62 artículos auto-informativos de respuestas verdadero y falso. Luego de ser aplicado un análisis factorial el inventario adaptado para Chile quedo formado por 55 enunciados distribuidos en cuatro factores que son: a) Procesamiento elaborativo: está compuesto por 8 proposiciones, los cuales miden que la habilidad para personalizar, concretar y visualizar información es más importante que la habilidad más académica de abstraer; b) Estudio metódico: la principal estrategia de aprendizaje de los estudiantes que poseen este factor es hacer lo que se les dice que hagan y repetir la información tan a menudo como puedan antes del examen; c) Procesamiento profundo: los estudiantes que obtienen altos puntajes en esta escala son conceptuales, categorizan, evalúan críticamente lo apropiado de sus categorizaciones esto, demuestra que poseen un alto grado de habilidad de pensamiento crítico atienden más al significado de lo que estudian que a los aspectos superficiales. Las asociaciones conceptuales significan la última etapa en el continuo del procesamiento; d) Retención de hechos: está asociada con la tendencia a categorizar información en categorías estrechas y precisas.

Para el análisis de datos se utilizaron estadísticos descriptivos como media, desviación estándar (DE) y porcentajes para las variables del estudio (tipo de liceo, sexo y ubicación urbana o rural del liceo) con cada uno de los factores del inventario y los estadísticos inferenciales de análisis ANOVA de un factor para comparar los resultados según los tipos de enseñanza y prueba t de Student para comparar los puntajes en el inventario entre sexo y área geográfica. Para realizar todos los análisis se utilizó el Software estadístico Statistical Package for the Social Sciences SPSS versión 11.5. 


\section{RESULTADOS}

En la Tabla 1 podemos observar, que los estudiantes de liceos técnicos profesionales muestras utilizan principalmente las estrategias de aprendizaje de estudio metódico, procesamiento profundo y retención de hechos y un porcentaje menor la de procesamiento elaborativo.

\section{Tabla 1}

Porcentaje de estudiantes según tipo de liceo (técnico, científico humanista, politécnico y técnico agrícola) con respecto a tipo de estrategia de aprendizaje.

\begin{tabular}{|c|c|c|c|c|c|}
\hline Factor/categoría & $\begin{array}{c}\text { Baja } \\
(\%)\end{array}$ & $\begin{array}{c}\text { Media } \\
(\%)\end{array}$ & $\begin{array}{l}\text { Alta } \\
(\%)\end{array}$ & $\begin{array}{c}\text { Media } \\
\text { puntajes }\end{array}$ & $\mathrm{DE}$ \\
\hline \multicolumn{6}{|c|}{ Liceos técnicos profesionales } \\
\hline Procesamiento elaborativo & 59,6 & 32,1 & 8,3 & 4 & 1,6 \\
\hline Estudio metódico & 32,8 & 49,6 & 17,6 & 6,7 & 1,2 \\
\hline Procesamiento profundo & 22,8 & 50,4 & 26,8 & 9,4 & 1,5 \\
\hline Retención de hechos & 20,5 & 50,4 & 29,1 & 9,2 & 1,6 \\
\hline \multicolumn{6}{|c|}{ Liceos científicos humanistas } \\
\hline Procesamiento elaborativo & 35,6 & 41,1 & 23,3 & 5,1 & 1,5 \\
\hline Estudio metódico & 39,5 & 34,1 & 26,4 & 7 & 1,3 \\
\hline Procesamiento profundo & 24,8 & 41,9 & 33,3 & 9,2 & 1,7 \\
\hline Retención de hechos & 25,6 & 52,7 & 21,7 & 8,9 & 1,5 \\
\hline \multicolumn{6}{|l|}{ Liceos politécnicos } \\
\hline Procesamiento elaborativo & 40,9 & 36,4 & 22,7 & 4,7 & 1,1 \\
\hline Estudio metódico & 27,2 & 56,8 & 34 & 7,6 & 1,3 \\
\hline Procesamiento profundo & 20,5 & 45,5 & 33,2 & 9,8 & 1,2 \\
\hline Retención de hechos & 45,5 & 34,1 & 20,4 & 8,1 & 1,6 \\
\hline \multicolumn{6}{|l|}{ Liceos técnicos agrícolas } \\
\hline Procesamiento elaborativo & 49,9 & 36,6 & 13,3 & 4,4 & 1,7 \\
\hline Estudio metódico & 23,3 & 60 & 16,6 & 7,6 & 1,4 \\
\hline Procesamiento profundo & 16,7 & 63,3 & 20,0 & 9,2 & 1,5 \\
\hline Retención de hechos & 26,6 & 51,7 & 21,7 & 8,6 & 1,8 \\
\hline
\end{tabular}

Siguiendo en la Tabla 1, se puede ver que los estudiantes de liceos científicos humanistas utilizan principalmente las estrategias de retención de hechos, seguida por procesamiento profundo y elaborativo y en una menor medida utilizan 
la estrategia de procesamiento metódico. Con respecto a los liceos politécnicos, se puede observar que los estudiantes muestran una concentración mayor en estudio metódico y por el contrario se observa que el menor porcentaje se encuentra en la estrategia retención de hechos. Finalmente, la Tabla 1 muestra que los estudiantes de los liceos técnicos agrícolas tienen una agrupación mayor en procesamiento profundo, metódico y retención de hechos y menor en el procesamiento elaborativo.

Al evaluar si existen diferencias estadísticamente significativas de las estrategias de aprendizaje según tipo de liceos, solo se presenta en el procesamiento elaborativo $(p=0,0000)$, obteniendo una diferencia de un punto entre el puntaje mayor que fue para los liceos científico humanista (media $=5,1$, $\mathrm{DE}=1,5$ ) y el menor, que fue para los liceos técnicos profesionales (media $=4$, $\mathrm{DE}=1,6)$. Para el caso de los liceos politécnicos la media fue de 4,7 (DE =1,1) y para los liceos técnicos agrícola fue de 4,4 (DE =1,7).

En la Tabla 2, se puede observar que tanto las estudiantes mujeres como los hombres presentan una predominancia del procesamiento profundo, retención de hechos y estudio metódico, sin embargo, los hombres tienden a tener una menor predominancia del procesamiento elaborativo.

\section{Tabla 2}

Porcentajes de estudiantes según sexo y tipo de estrategia de aprendizaje.

\begin{tabular}{lccccc}
\hline Factor/categoría & $\begin{array}{c}\text { Baja } \\
(\%)\end{array}$ & $\begin{array}{c}\text { Media } \\
(\%)\end{array}$ & $\begin{array}{c}\text { Alta } \\
(\%)\end{array}$ & $\begin{array}{c}\text { Media } \\
\text { puntajes }\end{array}$ & DE \\
\hline Mujeres & & & & & \\
\hline Procesamiento elaborativo & 39,1 & 39,6 & 21,3 & 5 & 1,3 \\
\hline Estudio metódico & 27,2 & 44,4 & 28,4 & 8 & 1,4 \\
\hline Procesamiento profundo & 24,8 & 46,2 & 29 & 9,1 & 1,5 \\
\hline Retención de hechos & 30,2 & 46,1 & 23,7 & 8,6 & 1,4 \\
\hline Hombres & & & & & \\
\hline Procesamiento elaborativo & 54,3 & 33,8 & 11,9 & 4 & 1,2 \\
\hline Estudio metódico & 38 & 48,7 & 13,3 & 7 & 1,4 \\
\hline Procesamiento profundo & 20 & 51,3 & 28,7 & 9,5 & 1,5 \\
\hline Retención de hechos & 23,1 & 52,3 & 24,6 & 9,5 & 1,4 \\
\hline
\end{tabular}


Al comparar los puntajes, se puede observar diferencias estadísticamente significativas entre hombres y mujeres en el procesamiento elaborativo $(p=0,001)$ y el estudio metódico $(p=0,002)$, por lo tanto podemos decir que las estudiantes mujeres utilizaría mayormente el procesamiento elaborativo (media $=5, D E=1,3$ ) y estudio metódico (media $=8, D E=1,4$ ) en relación a los estudiantes hombres ( media $=4, \mathrm{DE}=1,2 ;$ media $=7, \mathrm{DE}=1,4$ respectivamente).

Por otro lado, si se compara dentro del grupo de mujeres según tipo de enseñanza, no se observan diferencias estadísticamente significativas, pero estas diferencias si se observan entre las estudiantes mujeres según liceo urbano y rural $(p=0,002)$, donde sólo el estudio metódico se utiliza más en estudiantes mujeres de liceos rurales (media $=8,8, D E=1,3$ ) que en las mujeres de liceos urbanos (media $=7,2, D E=1,4)$. Esto es interesante ya que la mayor parte de las mujeres de la muestra general pertenecen a liceos urbanos.

Por otro lado, al comparar en el grupo de estudiantes hombres, se puede decir que tanto para el factor procesamiento elaborativo $(p=0,002)$, como para el factor estudio metódico $(p=0,034)$ se aprecian diferencias significativas entre los grupos, por lo que se puede apreciar que los liceos científicos humanistas en el procesamiento elaborativo (media $=5, D E=1,2$ ) y los liceos técnicos agrícolas en el estudio metódico (media $=8, D E=1,6$ ) se inclinan hacia estas estrategias. $\mathrm{El}$ el grupo de estudiantes hombres no se observaron diferencias significativas con respecto a la ubicación urbana o rural de los liceos.

En la Tabla 3, se puede observar que un mayor porcentaje de estudiantes de liceos urbanos presentan las estrategias de aprendizaje de procesamiento profundo, estudio metódico y retención de hechos y, en menor porcentaje, el procesamiento elaborativo. En el caso de los liceos rurales, se evidencia un menor uso de la estrategia de estudio metódico. 
Tabla 3

Porcentajes de estudiantes según ubicación geográfica (urbano y rural) y tipo de estrategia de aprendizaje.

\begin{tabular}{lccccc}
\hline Factor/categoría & $\begin{array}{c}\text { Baja } \\
(\%)\end{array}$ & $\begin{array}{c}\text { Media } \\
(\%)\end{array}$ & $\begin{array}{c}\text { Alta } \\
(\%)\end{array}$ & $\begin{array}{c}\text { Media } \\
\text { puntajes }\end{array}$ & DE \\
\hline Urbano & & & & & \\
\hline Procesamiento elaborativo & 48,4 & 36,5 & 15,1 & 4,5 & 1,4 \\
\hline Estudio metódico & 34,9 & 50,9 & 14,5 & 6,8 & 1,2 \\
\hline Procesamiento profundo & 21,4 & 51,3 & 27,3 & 9,3 & 1,8 \\
\hline Retención de hechos & 26,3 & 50,2 & 24,4 & 8,8 & 1,7 \\
\hline Rural & & & & & \\
\hline Procesamiento elaborativo & 43 & 36,7 & 20,3 & 4,9 & 1,2 \\
\hline Estudio metódico & 26,6 & 31,6 & 41,8 & 8,1 & 1,3 \\
\hline Procesamiento profundo & 25,2 & 40,6 & 34,2 & 9,3 & 1,6 \\
\hline Retención de hechos & 30,4 & 49,5 & 24,2 & 8,9 & 1,4 \\
\hline
\end{tabular}

Al comparar de los puntajes de las estrategias de aprendizaje según liceo urbano o rural, solo existen diferencias estadísticamente significativas con la estrategia de estudio metódico Esto se refleja en las diferencias de medias que alcanzan un punto mayor en el área geográfica rural (media $=8,1, D E=1,3$ ) con respecto al área urbana (media $=6,8, \mathrm{DE}=1,2$ ) ; por lo tanto podemos decir que los estudiantes de liceos rurales utilizarían mayormente esta estrategia en comparación a los estudiantes de liceos urbanos.

Con respecto a la comparación dentro del grupo urbano, se observa que el análisis arrojó que existen diferencias significativas entre la variable tipos de enseñanza y la estrategia procesamiento elaborativo $(p=0,015)$, estudio metódico $(p=0,002)$ y retención de hechos $(p=0,026)$. Esto se puede apreciar si comparamos las medias obtenidas por los estudiantes según tipo de enseñanza; en donde se puede estimar que en cuanto al procesamiento elaborativo los estudiantes de los liceos científicos humanistas obtienen el mayor puntaje (media $=6, D E=1,3$ ), lo que expresaría que dentro del grupo urbano son los que mayormente utilizan ésta estrategia. Por otra parte se puede ver que los estudiantes de los liceos técnicos agrícolas urbanos son los que más utilizan el estudio metódico (media $=7,6, \mathrm{DE}$ $=1,5$ ) en comparación a los estudiantes de otros liceos. Por último se puede observar que los estudiantes de liceos técnicos profesionales urbanos utilizan principalmente la estrategia de retención de hechos ( media $=9,3, \mathrm{DE}=1,2$ ). 
Con respecto al tipo de enseñanza de estudiantes rurales, análisis arrojó que existen diferencias significativas para el factor procesamiento elaborativo $(p=0,019$ ). Si comparamos las medias podemos decir que los estudiantes de los liceos de tipo de enseñanza científicos humanistas (media $=5,2, \mathrm{DE}=1,7$ ) en comparación a los otros tipos de enseñanzas son los que más utilizan esta estrategia.

\section{DISCUSIÓN}

Al describir las estrategias de aprendizajes de los estudiantes de tercer año medio de liceos municipales de la provincia de Talca, según las distintas variables propuestas para la investigación, se observa, qué existe una utilización media de las estrategias de aprendizajes para cada uno de los tipos de enseñanza, área geográfica y sexo de la muestra total.

Es importante mencionar que existen pequeñas diferencias a nivel descriptivo con respecto a las variables, y el uso de las estrategias, las que podrían ser explicadas, debido a las diferentes asignaturas que presentan los liceos utilizados en la muestra, como lo plantea Salinas (2004), el cual afirma que ciertos tipos de educación están basados en contenidos de las llamadas "ciencias duras" 0 exactas, por lo que favorecerían más el uso de una estrategia con respecto a otras. Otra implicancia en estas diferencias puede deberse a que las estrategias de estudio metódico, procesamiento elaborativo y procesamiento profundo se vinculan entre sí, y estas de forma negativa con retención de hechos (Truffelo y Pérez, 1998).

Al comparar las estrategias de aprendizajes de los estudiantes de tercer año medio de liceos municipales de la provincia de Talca, con respecto al sexo, tipo de enseñanza y área geográfica, se evidenció que existe diferencia en las estrategias de aprendizajes para cada una de las variables, en cuanto al sexo, las diferencias significativas se manifiestan en las estrategias procesamiento elaborativo y estudio metódico, siendo el grupo femenino aquel que marca una diferencia mayor en ambas estrategias. Esto coincide con lo planteado por Cano (2000) quien dice que existe una relación significativa entre el sexo de los estudiantes y el despliegue de sus estrategias y estilos de aprendizajes.

En cuanto al tipo o modalidades de enseñanza las diferencias se presentan con respecto a la estrategia de aprendizajes procesamiento elaborativo, en donde 
los liceos científicos humanistas y técnicos profesionales son los que más difieren para dicha estrategia. Esto se pudiese entender debido a que los liceos técnicos profesionales se encuentran regulados por el tipo de carrera que impartan, por ello se puede presumir que la estrategia que utilicen estará modulada por ello (Cano 2000).

En relación al área geográfica (rural-urbano) existen diferencias respecto a la estrategia estudio metódico; mostrándose una mayor tendencia a utilizar ésta estrategia en el grupo rural. Esto coincide con los resultados obtenidos respecto al porcentaje de estudiantes de esta área, los que puntúan desde el nivel medio a alto en esta estrategia; por lo tanto se puede decir que estas diferencias en dicha estrategia serían explicadas debido a que el número de mujeres es mayor en el conglomerado rural en relación al grupo masculino para este mismo. Complementando esto, existe evidencia de que el estudio sistemático es mayor en el caso de las mujeres, quienes tienden a ser más esquematizadas y organizadas que los varones (Zamora et al, 2004).

Al comparar las estrategias de aprendizajes de los estudiantes de tercer año medio de liceos municipales y las variables sexo, tipo de enseñanza y área geográfica se puede decir que la mayoría de las estrategias muestran diferencias significativas con respecto a las variables antes mencionadas. La variable sexo se diferencia en el procesamiento elaborativo y metódico, en cuanto al tipo de enseñanza se distinguen todas las estrategias, salvo procesamiento profundo. En el área geográfica, la diferencia sólo se muestra para estudio metódico y procesamiento profundo.

La estrategia retención de hechos sólo vincula con una de las variables, que es tipo de enseñanza, esto puede tener relación con que el tipo de enseñanza apunta directamente a los contenidos que el estudiante debe asimilar. Es relevante mencionar que en todas las variables existe una relación con el estudio sistemático por lo que nos lleva a pensar que esta estrategia puede ser desarrollada de mejor forma en los estudiantes.

En todo caso, se considera que los hallazgos de este estudio es un aporte valioso para los establecimientos de enseñanza media o secundaria, ya que puede permitir comprender de alguna forma las diferencias en cuanto al uso y manejo de las estrategias de aprendizajes, así como también las posibles formas de mejorarlas, y de este modo contribuir al uso de nuevas prácticas pedagógicas, buscando nuevas metodologías que estimulen el reconocimiento por parte de los 
estudiantes de la estrategia que utilizan con mayor frecuencia creando "consciencia" de la importancia de éstas, no sólo para obtener un mejor rendimiento, sino para un mayor entendimiento de las materias difundidas por el docente. Es fundamental que el estudiante conozca la estrategia que más le acomoda para aprender y de esta forma emplearla adecuadamente en el aula.

Por otra parte al ser un estudio a nivel local, nos permite tener antecedentes nuevos y recientes respecto al tema de las estrategias en los estudiantes de la provincia de Talca, lo que puede ser un aporte al conocer las distintas estrategias empleadas en los liceos, lo cual permitiría tener un acercamiento mayor hacia como se hace significante la enseñanza dentro del aula, y como potenciar lo que los estudiantes aprender.

Esta investigación presenta ciertas limitaciones, su diseño al ser de naturaleza transversal, recogió los datos a través de cuestionarios en un momento del tiempo por lo que no se sabe con certitud el origen de las diferencias observadas entre los estudiantes; en éste sentido creemos que además es relevante considerar que al presentarnos ante los estudiantes pudimos causar en ellos ansiedad o necesidad de logro, lo qué pudo interferir en los resultados.

Por todo lo anterior es relevante mencionar que los datos obtenidos en este estudio sean tomados como pruebas iníciales y no concluyentes debido que los datos puedan estar influidos por las características de la muestra, el procedimiento empleado y la aplicación de éste. Es importante seguir estudiando las estrategias de aprendizajes y las variables género, tipo de enseñanza y área geográfica, que creemos han sido de utilidad para conocer perspectivas distintas a las generalidades estudiadas como lo es el rendimiento académico en relación con el género, por nombrar algunas. Sin embargo, sería interesante hacer un trabajo en conjunto con las variables expuestas y su complemento con datos más específicos o personales del estudiante, para de este modo contribuir de un modo más preciso y verás a la información que por siempre será tema de estudio, cómo lo son las "estrategias de aprendizajes".

Otra línea de investigación que sería importante revisar es la que corresponde a la intervención de los docentes, tanto en la adquisición como en el desarrollo adecuado y permanente de las estrategias al momento de recoger la información, pues de esta forma el estudiante podría automotivarse y, por ende, rendir mejor académicamente. 
Para esto es crucial identificar y practicar estrategias en el aula; una forma de hacerlo sería utilizar cuestionarios respecto a: ¿Cómo lo hacen? (para entender o aprender una materia) ¿Qué pasos han seguido que les resultan, y cuáles no? (para que el estudiante reflexione acerca de las ventajas y desventajas de los métodos hasta ahora utilizados), pues ello ayudaría a fortalecer la experiencia de aprender en el liceo.

\section{REFERENCIAS BIBLIOGRÁFICAS}

BADENIER, C. (2003). Confiabilidad y validez del Learning and Study Strategies inventory (LASSI) en una muestra de estudiantes de la región metropolitana. Psykhe, 12, 193-206.

BERMEOSOLO, J. (2005). Como aprenden los seres humanos mecanismos psicológicos del aprendizaje. Santiago de Chile: Ediciones Universidad Católica de Chile.

CABRERA, J., FARIÑAS, G. (2005). El estudio de los estilos de aprendizajes desde una perspectiva Vigostkiana: una aproximación conceptual, Revista Iberoamericana de Educación (ISSN: 1681-5653).

CÁRDENAS, B. (2006). Hacia el desarrollo de la habilidad de la expresión escrita y sus implicaciones didácticas en el proceso de adquisición del idioma español como segunda lengua. Tesis para aspirar al grado de doctor en aportaciones educativas en Ciencias Sociales y Humanas, Departamento de Método de Investigación y Diagnostico en Educación, Universidad de Granada, Granada, España.

CARRASCO, J., STINGO, M. Y LAVAL, E. (2001). Informática educativa para las escuelas rurales en Chile. Temuco: Universidad de la Frontera, Instituto de Informática Educativa.

GÁZQUEZ, J., PÉREZ, M., RUIZ, M., MIRAS, F.\& VICENTE, F. (2006). Estrategias de aprendizajes en estudiantes de enseñanza secundaria obligatoria y su relación con la autoestima. International Journal of Psychology and Psichocological Therapy, 1, 51-62. 
GUANIPA, M., MOGOLLÓN, E. (2006). Estilos de aprendizajes y estrategias cognitivas en estudiantes de ingeniería, Revista Ciencias de la Educación, 27, 11-27.

MASSONE, A., GONZÁLEZ, G. (2003). Analisis del uso de estrategias cognitivas de aprendizaje, en estudiantes de noveno año de educación general básica [versión electrónica]. Revista Iberoamericana de Educación, 1-5. Extraído el 7 de enero, 2008, de http://www.rieoli.org/deloslectores/551massone.pdf

MUÑOZ, M. (2005). Estrategias de aprendizajes en estudiantes universitarias [versión electrónica] Psicología Científica.com, 1-11. Extraído el 10 de agosto, 2007, de http://www.psicologiacientifica.com/bv/psicologia-62-1-estrategias-deaprendisaje-en-estudiantes-universitarias.html

TRUFFELLO, I. Y PÉREZ, F. (1998). Diseño y evaluación de actividades instruccionales conducentes a las estrategias de aprendizaje elaborativo y profundo. Revista Enfoques Educacionales, 1, 1-12.

PIZANO, G. (2004). Las estrategias de aprendizaje y su relevancia en el rendimiento académico de los alumnos. Revista de Investigación Educativa, 14, 27-30.

ROUX, R. Y ANZURES, E. (2014). Estrategias de aprendizaje y su relación con el rendimiento académico en estudiantes de una escuela privada de educación media superior. Revista Actualidades Investigativas en Educación, 15, 1-16.

SALINAS, H. (2004). Uso de internet como herramienta pedagógica para facilitar el aprendizaje elaborativo y profundo. Tesis para optar al grado de Magister en Educación, mención Informática Educativa. Escuela de Postgrado Facultad de Ciencias Sociales, Universidad de Chile, Santiago, Chile.

ZAMORA, M., RUBILAR, F. \& RAMOS, H. (2004). Estudio descriptivo de las estrategias cognitivas y metacognitivas de los alumnos y alumnas de primer año de pedagogía en enseñanza media de la universidad del Bío-Bío, Theoria, vol.13, 103-110.

ZULIA, C. (2006). Estrategias de aprendizaje cognitivas y Metacognitivas activadas por los estudiantes de enfermería en la asignatura de farmacología. Trabajo de grado para optar a la Maestría en Educación, mención: Educación Superior. Línea de investigación en ciencias cognitivas, en Instituto Pedagógico "Rafael Alberto Escobar Lara", Maracay, Venezuela. 